\title{
Introduction to the Special Issue on Computer Modelling of Transmission, Spread, Control and Diagnosis of COVID-19
}

\author{
Yudong Zhang ${ }^{1, *}$, Qilong Wang ${ }^{2}$ and Sean H. Y. Yuan ${ }^{3}$ \\ ${ }^{1}$ School of Informatics, University of Leicester, Leicester, LE1 7RH, UK \\ ${ }^{2}$ The Affiliated Huai'an No. 1 People's Hospital, Nanjing Medical University, Huai'an, 223300, China \\ ${ }^{3}$ Department of Biomedical Sciences, City University of Hong Kong, Hong Kong, China \\ ${ }^{*}$ Corresponding Author: Yudong Zhang. Email: yudongzhang@ieee.org \\ Received: 02 March 2021 Accepted: 10 March 2021
}

The 2019-20 coronavirus pandemic is an ongoing pandemic of coronavirus disease 2019 (COVID-19) caused by severe acute respiratory syndrome coronavirus 2 (SARS-CoV-2). It was declared to be a Public Health Emergency of International Concern and recognized as a pandemic by the World Health Organization. The outbreak of COVID-19 has rapidly spread to most countries in the world. This special issue started at 21 April 2020, when there were more than 2.5 million confirmed cases and 174,336 deaths around the world. After 10 months, this special issue closes at 23 February 2021, the two figures increase to 112.2 million confirmed cases and 2.48 million death tolls, respectively.

Computer modelling (CM) plays an important role in fighting COVID-19. For example, CM techniques can help develop vaccine \& targeted drugs for COVID-19. In epidemiology, CM can provide tracking and prediction of the spread speed of infected territories and areas, so as to assist policymakers to make appropriate decisions. The visualization technology provides a global overview for policymakers. AI and CM methods are efficient in making fast and accurate diagnoses of COVID-19 using trained models, based on routine CT or X-ray or other imaging tools. Wearable sensors can monitor abnormality for home-based mild COVID-19 patients. Human behavioral data can be analyzed to make better individual or community quarantine and social control policies. CM can help predict the protein structures of the coronavirus. The emotional data in mental health can be utilized to help people to cope with self-quarantined people. CM can manage medical resources (e.g., face masks, ventilator, et al.) supply chain.

A total of 40 manuscripts were submitted and 11 were selected based on a robust peerreviewed process. The 11 articles are authored by researchers from world-wide universities, and reflect state of the research developments and initiatives in the computer modelling of transmission, spread, control and diagnosis of COVID-19.

The first paper "Estimating the Impact of COVID-19 Pandemic on the Research Community in the Kingdom of Saudi Arabia" by Attaallah et al. [1] executes the forecasting model for predicting the possible number of COVID-19 cases in Saudi Arabia till a defined period.

The second paper "Prediction of Proteins Associated with COVID-19 Based Ligand Designing and Molecular Modeling" by Monajjemi et al. [2] has accomplished docking studies on the major

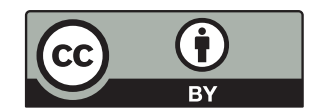

This work is licensed under a Creative Commons Attribution 4.0 International License, which permits unrestricted use, distribution, and reproduction in any medium, provided the original work is properly cited. 
virus protease with 4 natural product species as anti COVID-19 (SARS-CoV-2) which have been extracted from Gillan's leaves plants.

In the third paper entitled "Predictive Models for Cumulative Confirmed COVID-19 Cases by Day in Southeast Asia" by Areepong et al. [3], the authors investigate the suitable forecasting model for the number of infectious people in Southeast Asian countries. They also presented a comparison of forecasting models between logistic growth curve and Gompertz growth curve.

In their paper "Real-Time Analysis of COVID-19 Pandemic on Most Populated Countries Worldwide", Gupta et al. [4] make a comparative analysis of the top 5 most populated countries namely United States, India, China, Pakistan and Indonesia, from 1st January 2020 to 31 st July 2020. The authors also target to predict an increase in the number of deaths and total infected cases in these five countries.

In their paper "Study of Non-Pharmacological Interventions on COVID-19 Spread," Singh et al. [5] proposed three different mathematical models with different scenarios, such as screening, quarantine, and NPIs, to estimate the number of virus spreaders.

The sixth paper "SEIHCRD Model for COVID-19 Spread Scenarios, Disease Predictions and Estimates the Basic Reproduction Number, Case Fatality Rate, Hospital, and ICU Beds Requirement" by Singh et al. [6] proposes a new mathematical method, the SEIHCRD model, which has an excellent potential to predict the incidence of COVID-19 diseases. This proposed SEIHCRD model is an extension of the SEIR model.

In the seventh paper "Modelling the Effect of Self-Immunity and the Impacts of Asymptomatic and Symptomatic Individuals on COVID-19 Outbreak" written by Biswas et al. [7], the authors reveal that the death rate from coronavirus disease increases as the infection rate increases, whereas infection rate extensively decreases with the increase of quarantined individuals.

Zuber et al. [8], in their paper "Investigation of Coronavirus Deposition in Realistic Human Nasal Cavity and Impact of Social Distancing to Contain COVID-19: A Computational Fluid Dynamic Approach," use Computational Fluid Dynamics as a solution tool to investigate the impact of COVID-19 deposition (i) On a realistic 3D human upper airway model and (ii) 2D social distancing protocol for a distance of $0.6,1.2,1.8$, and $2.4 \mathrm{~m}$.

Among all the possible applications, Al-Marzouki et al. [9] in their paper "Topp-Leone Odd Fréchet Generated Family of Distributions with Applications to COVID-19 Data Sets" consider two data sets of current interest, linked to the COVID-19 pandemic. They concern daily cases confirmed and recovered in Pakistan from March 24 to April 28, 2020. Their proposed model has the best fitting results in comparison to serious challengers, including the former odd Fréchet model.

In the tenth paper entitled "Validating the Correct Wearing of Protection Mask by Taking a Selfie: Design of a Mobile Application "CheckYourMask" to Limit the Spread of COVID-19," Hammoudi et al. [10] propose a design of a mobile application for permitting everyone having a smartphone and being able to take a picture to verify that his/her protection mask is correctly positioned on his/her face.

Finally, Sethi et al. [11] devise a highly accurate and real-time technique that can efficiently detect non mask faces in public and thus enforcing to wear mask, in their paper "A Real-time Integrated Face Mask Detector to Curtail Spread of Coronavirus.”

As a final remark, we wish this special issue can contribute to the fight against COVID-19 and it may benefit broader readers of researchers, practitioner, and students who are interested 
in related topics. We expect all readers can keep hope, resilience, and inspiration during this pandemic.

Acknowledgement: We would like to thank the authors for their contributions to this Special Issue. We also thank the journal of CMES for their financial supports for publications of this Special Issue, since all fees are waived for this special issue.

Funding Statement: This editorial work was partially supported by Hope Foundation for Cancer Research, UK (RM60G0680); British Heart Foundation Accelerator Award, UK; Royal Society International Exchanges Cost Share Award, UK (RP202G0230); Medical Research Council Confidence in Concept Award, UK (MC_PC_17171).

Conflicts of Interest: The authors declare that they have no conflicts of interest to report regarding the present study.

\section{References}

1. Attaallah, A., Ahmad, M., Seh, A. H., Agrawal, A., Kumar, R. et al. (2021). Estimating the impact of COVID-19 pandemic on the research community in the Kingdom of Saudi Arabia. Computer Modeling in Engineering \& Sciences, 126(1), 419-436. DOI 10.32604/cmes.2021.014263.

2. Monajjemi, M., Esmkhani, R., Mollaamin, F., Shahriari, S. (2020). Prediction of proteins associated with COVID-19 based ligand designing and molecular modeling. Computer Modeling in Engineering \& Sciences, 125(3), 907-926. DOI 10.32604/cmes.2020.012846.

3. Areepong, Y., Sunthornwat, R. (2020). Predictive models for cumulative confirmed COVID-19 cases by day in Southeast Asia. Computer Modeling in Engineering \& Sciences, 125(3), 927-942. DOI 10.32604/cmes.2020.012323.

4. Gupta, M., Jain, R., Gupta, A., Jain, K. (2020). Real-time analysis of COVID-19 pandemic on most populated countries worldwide. Computer Modeling in Engineering \& Sciences, 125(3), 943-965. DOI 10.32604/cmes.2020.012467.

5. Singh, A., Chandra, S. K., Bajpai, M. K. (2020). Study of non-pharmacological interventions on COVID-19 spread. Computer Modeling in Engineering \& Sciences, 125(3), 967-990. DOI 10.32604/cmes.2020.011601.

6. Singh, A., Bajpai, M. K. (2020). SEIHCRD model for COVID-19 spread scenarios, disease predictions and estimates the basic reproduction number, case fatality rate, hospital, and ICU beds requirement. Computer Modeling in Engineering \& Sciences, 125(3), 991-1031. DOI 10.32604/cmes.2020.012503.

7. Biswas, M. H. A., Islam, M. A., Akter, S., Mandal, S., Khatun, M. S. et al. (2020). Modelling the effect of self-immunity and the impacts of asymptomatic and symptomatic individuals on COVID-19 outbreak. Computer Modeling in Engineering \& Sciences, 125(3), 1033-1060. DOI 10.32604/cmes.2020.012792.

8. Zuber, M., Corda, J. V., Ahmadi, M., Shenoy, B. S., Badruddin, I. A. et al. (2020). Investigation of coronavirus deposition in realistic human nasal cavity and impact of social distancing to contain COVID-19: A computational fluid dynamic approach. Computer Modeling in Engineering \& Sciences, 125(3), 11851199. DOI 10.32604/cmes.2020.015015.

9. Al-Marzouki, S., Jamal, F., Chesneau, C., Elgarhy, M. (2020). Topp-Leone odd fréchet generated family of distributions with applications to COVID-19 data sets. Computer Modeling in Engineering \& Sciences, 125(1), 437-458. DOI 10.32604/cmes.2020.011521.

10. Hammoudi, K., Cabani, A., Benhabiles, H., Melkemi, M. (2020). Validating the correct wearing of protection mask by taking a selfie: Design of a mobile application "CheckYourMask" to limit the spread of COVID-19. Computer Modeling in Engineering \& Sciences, 124(3), 1049-1059. DOI 10.32604/cmes.2020.011663.

11. Sethi, S., Kathuria, M., Kaushik, T. (2021). A real-time integrated face mask detector to curtail spread of coronavirus. Computer Modeling in Engineering \& Sciences, 127(2), 389-409. 